\title{
Intervenção breve aplicada a universitários consumidores de risco de bebidas alcoólicas*
}

\author{
Wanda Cristina Sawicki ${ }^{1}$ \\ Dayana Souza Fram ${ }^{1}$ \\ Angélica Gonçalves Silva Belasco ${ }^{1}$
}

\begin{abstract}
Objetivo: investigar o consumo de álcool entre universitários de enfermagem e avaliar intervenção breve para abusadores de álcool. Método: estudo longitudinal, experimental. Dados coletados por questionário autoaplicável e três instrumentos. Análise estatística descritiva e utilização de testes específicos. Resultados: participaram 36 universitários e as drogas mais utilizadas foram: álcool (vodka, cerveja), tabaco e maconha. Idade do primeiro uso de álcool: 15 anos; repercussões após uso: quedas, cefaleia, vômitos e amnésia; e os motivos para consumo: social, sensação e prazer. Intervenção breve diminuiu significativamente o consumo de risco entre os abusadores. Entre os bebedores de baixo risco, metade referiu estar preparada para diminuir o consumo. Conclusão: intervenção breve diminui significativamente o padrão de consumo de álcool, promovendo a saúde.
\end{abstract}

Descritores: Estudantes; Enfermagem; Álcool; Promoção da Saúde.

\footnotetext{
* Artigo extraído da tese de doutorado "Qualidade de vida de graduandos de enfermagem e sua relação com o consumo de bebidas alcoólicas - intervenção breve para prevenção do consumo excessivo de álcool" apresentada à Escola Paulista de Enfermagem, Universidade Federal de São Paulo, São Paulo, SP, Brasil.

1 Universidade Federal de São Paulo, Escola Paulista de Enfermagem, São Paulo, SP, Brasil.
} 


\title{
Brief intervention applied to college students screened positive for at-risk alcohol consumption
}

Objective: to investigate the alcohol consumption among nursing college students and evaluate a brief intervention for abusers of alcoholic beverages. Method: longitudinal, experimental study. Data obtained through a self-applicable questionnaire and three instruments. Descriptive statistical analysis and use of specific tests. Results: 36 college students participated in this study. The most used drugs were: alcohol (vodka, beer), tobacco and marijuana. Age of first contact with alcoholic beverages: 15 years; repercussions after use: falls, headache, vomiting and amnesia; and the reasons for consumption: socialization, sensation and pleasure. The brief intervention significantly decreased the risk of consumption among those who abused alcoholic beverages. Among low-risk alcohol consumers, half of them said they were ready to reduce consumption. Conclusion: the short intervention significantly decreased the pattern of alcohol consumption thus promoting the health of the students.

Descriptors: Students; Nursing; Alcohol; Health Promotion.

\section{Intervención breve aplicada a universitarios consumidores de riesgo de bebidas alcohólicas}

\begin{abstract}
Objetivo: investigar el consumo de alcohol entre universitarios de enfermería y evaluar la intervención breve para los abusadores de alcohol. Método: estudio longitudinal, experimental. Los datos recolectados por cuestionario autoaplicable y tres instrumentos. Análisis estadístico descriptivo y uso de pruebas específicas. Resultados: participaron 36 universitarios, las drogas más utilizadas fueron alcohol (vodka, cerveza), seguido de tabaco y marihuana. La edad del primer uso de alcohol 15 años, repercusiones después del uso fueron caídas, cefalea, vómitos y amnesia y los motivos para el consumo: social, sensación y placer. La intervención breve disminuyó significativamente el consumo de riesgo entre los abusadores y entre los bebedores de bajo riesgo, la mitad, dijo estar dispuesta a disminuir el consumo. Conclusión: intervención breve disminuye significativamente el patrón de consumo de alcohol promoviendo la salud.
\end{abstract}

Descriptores: Estudiantes; Enfermería; Alcohol; Promoción de la Salud.

\section{Introdução}

Consumo abusivo e nocivo de álcool é um grave problema de saúde pública global, que causa consequências físicas e psicossociais e danos a outras pessoas do convívio ${ }^{(1-2)}$.

Segundo relatório da Organização Panamericana de Saúde (2012), o consumo médio de álcool nas Américas foi maior do que em outras partes do mundo. Em particular, as taxas de frequência de consumo excessivo ou beber pesado episódico (BPE) corresponderam a $13 \%$ entre mulheres e $29,4 \%$ entre homens. Em média, os adolescentes consumiram mais álcool, a cada vez que beberam, apesar de a frequência ser menor ${ }^{(2)}$.

O BPE corresponde ao consumo mínimo de 60 gramas, aproximadamente 5 doses típicas de álcool, pelo menos, uma vez nos últimos 30 dias, o que pode gerar risco de danos à saúde ${ }^{(2)}$. A Organização Mundial de Saúde (OMS) destaca a vulnerabilidade das mulheres para os malefícios do álcool e o aumento do consumo 
de álcool entre as mesmas como produto relacionado ao desenvolvimento econômico e mudança de papéis do gênero(1).

Estudo norte-americano desenvolvido com universitários de enfermagem identificou fatores de risco relacionados ao abuso do álcool, foram eles: falta de informação sobre álcool; expectativas sobre o curso que aumentavam o estresse e a ansiedade; hábitos de vida pouco saudáveis; isolamento social, influências individuais e dos pares. Como fatores de proteção, evidenciaram-se as políticas universitárias diferenciadas; experiências de vida; responsabilidades; e influências de colegas $^{(3)}$.

O Plano de Ações para Enfrentamento das Doenças Crônicas não Transmissíveis, estabelecido no Brasil, incluiu como meta a redução da prevalência do consumo nocivo de álcool, de 18\% para 12\% entre 2011 e 2022, visando impactar na redução de doenças cardíacas, hepáticas, mentais, oncológicas, acidentes e violência ${ }^{(4)}$.

A possibilidade da redução do uso abusivo do álcool depende de medidas de regulação adotadas pelos governos e do enfrentamento do poder das indústrias de álcool e associados ${ }^{(5)}$; destacando-se o aumento do preço das bebidas alcoólicas, a taxação de impostos e a proibição da publicidade relacionada ao incentivo do consumo de álcool, recomendado pela $\mathrm{OMS}^{(1)}$. Outra ação importante é a Intervenção Breve (IB), que apresenta como pressuposto teórico que comportamentos inadequados podem ser modificados com a utilização de estratégias educacionais, aconselhamento breve e entrevista motivacional( ${ }^{(6)}$.

A IB pode ser realizada por profissionais da saúde para prevenção do consumo excessivo de álcool como primeiro passo para motivar a reflexão sobre o padrão de consumo de álcool(7).

Os objetivos desta pesquisa foram identificar o consumo de substâncias psicoativas (SPA), o padrão de consumo de álcool e suas características entre universitários de enfermagem e o impacto da intervenção breve sobre o padrão de consumo de universitários classificados como bebedores de risco/nocivo/provável dependência.

\section{Método}

Estudo aprovado pelo Comitê de Ética em Pesquisa da Universidade Federal de São Paulo (UNIFESP) (CAAE: 40884915.9.0000.5505). Os participantes assinaram o Termo de Consentimento Livre e Esclarecido e foram garantidos o anonimato e a confidencialidade das informações.

Pesquisa longitudinal, experimental, realizada na Escola Paulista de Enfermagem da UNIFESP, entre março de 2015 e setembro de 2016. Após autorização da coordenação e dos professores, realizou-se a coleta de dados e IB nas salas de aula.

Participaram da pesquisa 281 universitários de todas as séries. Coletaram-se dados sociodemográficos e o padrão do consumo de álcool por meio do instrumento The Alcohol Use Disorders Identification Test (AUDIT), autorrelatado, validado no $\mathrm{Brasil}^{(8)}$, que avalia o padrão de consumo de álcool e rastreia uso problemático a partir do somatório de 10 questões, que varia de 0 a 40 pontos e classifica os consumidores de álcool em: baixo risco (0 a 7 pontos), uso de risco (8 a 15), uso nocivo (16 a 19) e provável dependência acima de 20 .

Após a coleta e tabulação dos dados, aplicou-se a

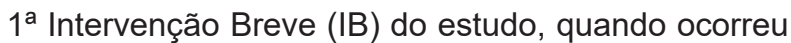
o feedback sobre a pontuação do AUDIT, para cada série e globalmente, seguida de palestra intitulada "Consumo de álcool e suas consequências"; entrega do folder e cartilha educativa encaminhada para o endereço eletrônico de cada série. Após a primeira IB, 41(14,59\%), universitários permaneceram classificados como abusadores de álcool e, destes, 36 continuaram no estudo e cinco desistiram.

Na segunda IB (um ano após a primeira), realizouse novo feedback do resultado do padrão de consumo excessivo que cada universitário apresentou, sendo reorientados para leitura da cartilha educativa e posteriormente solicitada avaliação da IB. Foram feitas questões sobre o perfil do consumo de outras substâncias psicoativas, aplicado o AUDIT e o instrumento The Drinker Inventory of Consequences (DrlnC) ${ }^{(9)}$, validado no Brasil, contém 50 questões subdivididas em 5 domínios (físico, interpessoal, intrapessoal, controle impulso e responsabilidade social) e avalia as consequências do consumo exagerado de álcool. As respostas são dicotômicas, o somatório gera escore total entre 0 e 45 pontos e as consequências do consumo exagerado de álcool são classificadas em: muito baixa (0-23), baixa (24-28), média (29-34), alta (35-38) e muito alta (39-45). Utilizou-se, ainda, a régua de prontidão para mudança (RPM) com o objetivo de verificar a motivação dos universitários para diminuir o padrão de consumo de álcool. A RPM é uma técnica analógico-visual, simples e eficaz, para averiguar qual é o grau de motivação para mudança de atitude por parte do sujeito. $\mathrm{O}$ avaliador pergunta qual dos pontos da régua melhor reflete o quão pronto ele está, no presente momento, para mudar seu comportamento-problema; a pontuação varia de zero a dez, considerando que zero indica que o sujeito não está pronto e dez que está ativamente trabalhando na mudança do seu comportamento(10).

Para tabulação dos dados, utilizou-se o programa Excel, 2010 e para os cálculos estatísticos, o Statistical 
Package for the Social Sciences (SPSS) IBM, versão 19. Para análise descritiva das variáveis contínuas, calcularam-se a média, desvio padrão, mediana, mínimo e máximo; para variáveis categóricas frequência e percentual. Para evolução do AUDIT e das variáveis específicas, o Generalized Estimating Equation (GEE); para comparações múltiplas, o Teste da Razão de Verossimilhança e nível de significância de $5 \%(p \leq 0,05)$.

\section{Resultados}

A idade média dos universitários foi 20 anos; 33 (91,7\%) mulheres, 28 (77,8\%) brancos; 5 (13,9\%) amarelos e $3(8,3 \%)$ pardos; todos solteiros; $16(44,4 \%)$ católicos, 11 (30,6\%) sem religião, 5 (13,9\%) espíritas e $4(11,1 \%)$ evangélicos. Cursavam a $1^{a}$ série $13(36,1 \%)$ estudantes, $2^{\mathrm{a}}$ série $9(25 \%)$, $3^{\mathrm{a}}$ série $9(25 \%)$ e $4^{\mathrm{a}}$ série 5 (13,9\%). A renda familiar declarada por 9 (25\%) foi de até 13 salários mínimos (SM); $12(33,3 \%)$ de até $7 ; 11(30,5 \%)$ até 4,$5 ; 2(5,6 \%)$ até $2 ; 1(2,8 \%)$ até 1,5 ; e $1(2,8 \%)$ até $1 \mathrm{SM}$. Dois (5,6\%) universitários trabalhavam, um como bar tendere outro como estagiário de enfermagem, com renda média de 1,5 SM.

Questionados sobre o consumo na vida de SPA, $26(72,2 \%)$, afirmaram tê-lo feito e quanto ao uso atual, exceto álcool, $8(22,2 \%)$ universitários referiram usar uma ou mais SPAe, destes, $7(87,5 \%)$ referiram tabaco, $3(37,5 \%)$ maconha e $1(12,5 \%)$ cocaína.

A idade mediana do $1^{\circ}$ uso de álcool foi 15 anos (3-18). Quanto a bebidas alcoólicas utilizadas (questão de múltipla escolha), foram: vodka 32 (88,9\%), cerveja
$31(86,1 \%)$, tequila $24(66,7 \%)$, saquê $17(47,2 \%)$ e vinho/pinga $12(33,3 \%)$. Os locais onde consumiam as bebidas: bares $34(94,4 \%)$, baladas $32(88,9 \%)$, casa de amigos $28(77,8 \%)$ e $19(52,8 \%)$ perto da universidade.

Como justificativas para o consumo de álcool, 16 $(44,5 \%)$ universitários consumiam por motivos sociais, $11(30,5 \%)$ por apreciar o sabor e $9(25 \%)$ pela sensação e prazer.

Os eventos vivenciados após uso de álcool foram: quedas 23 (63,9\%); vexame em local público 20 (55,6\%); carona com motorista alcoolizado $15(41,7 \%)$; transar sem camisinha/briga em bares/baladas 7 (19,4\%) e outros (dirigir alcoolizado, briga com familiares e queimaduras). Como consequências: cefaleia 34 (94,4\%); vômito/náusea 26 (72,2\%); amnésia 25 (69,4\%); situação embaraçosa 11 (30,6\%); rompimento de relacionamento amoroso $8(22,2 \%)$ e outros (falta a compromisso, desmaio, trauma ortopédico e coma alcoólico).

Os resultados do Drlnc, aplicado após a segunda IB, demonstraram que 36 (100\%) universitários apresentaram baixo risco para consequências do consumo exagerado de álcool em todos os domínios do questionário.

Referiram ter lido a cartilha educativa $32(88,9 \%)$ universitários, $16(44,4 \%)$ avaliaram como boa, 13 $(36,1 \%)$ como muito boa e $3(8,3 \%)$ como regular.

A Figura 1 apresenta os resultados do AUDIT que apontam os padrões de consumo de álcool dos universitários, antes, etapa 1; após a $1^{\mathrm{a}} \mathrm{IB}$, etapa 2; e após a segunda IB, etapa 3 .

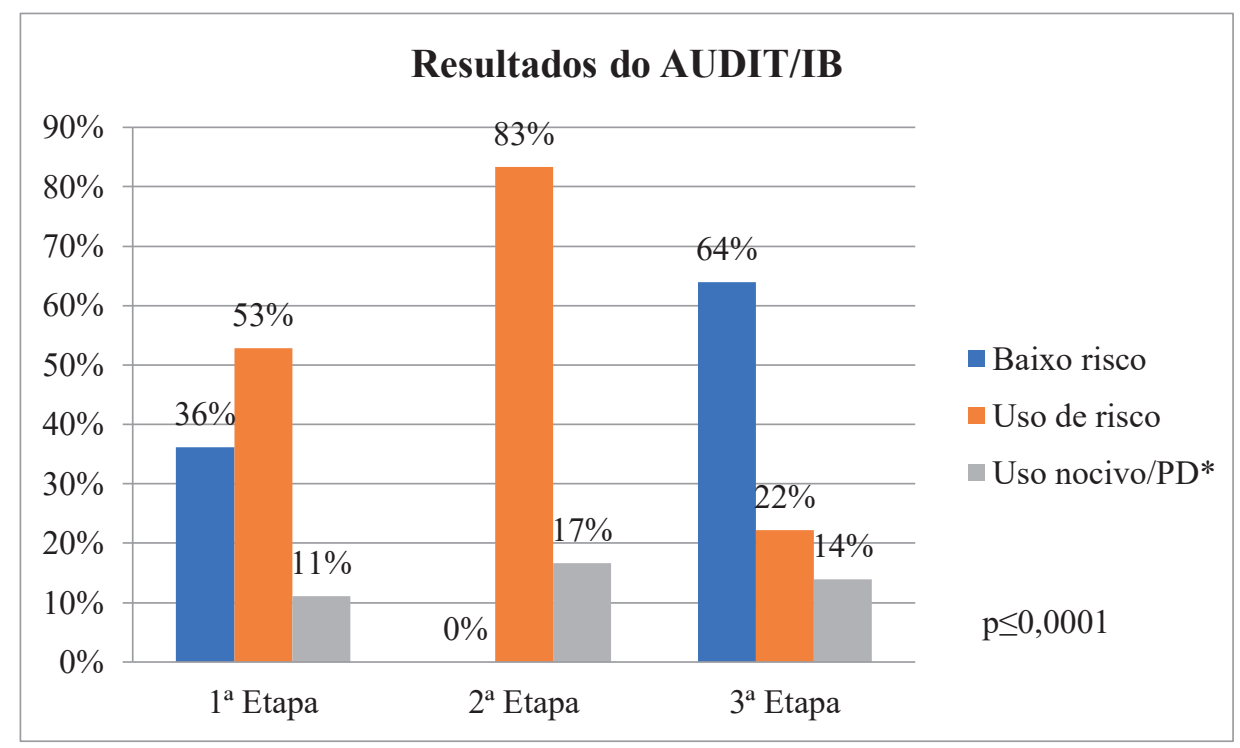

*PD: Provável dependência

Figura 1 - Comparação dos padrões de consumo de álcool, segundo o AUDIT, dos 36 universitários de enfermagem, antes e após cada IB. Escola Paulista de Enfermagem - UNIFESP. São Paulo, SP, Brasil, 2016 
A Tabela 1 mostra que houve correlação significante entre dimensões do AUDIT e algumas variáveis sociodemográficas e econômicas. Universitários da $1^{a}$ série apresentaram diminuição da frequência do consumo de 5 doses ou mais por episódio, passando a não consumir ou consumir menos de uma vez por mês, após a $2^{\mathrm{a}} \mathrm{IB}$, quando comparada às etapas anteriores do estudo $(p \leq 0,0086)$. Universitários da $3^{a}$ série consumiam mais doses do que os de outras séries $(p \leq 0,0054)$; os não brancos ingeriam mais doses $(p \leq 0,0335)$ e em maior frequência $(p \leq 0,0114)$ quando comparados aos brancos, mesmo após a $2^{\mathrm{a}} \mathrm{IB}$. Universitários com renda familiar de até 7 SM passaram a ingerir bebidas alcoólicas em menor frequência após a $2^{\mathrm{a}}$ IB $(p \leq 0,0427)$. Quanto menor a idade dos universitários, menor foi a frequência de ingesta de álcool após a $2^{\mathrm{a}} \mathrm{IB}(\mathrm{p} \leq 0,0001)$.

Tabela 1 - Grau de significância das correlações entre dimensões do AUDIT e variáveis sociodemográficas e econômicas dos 36 universitários de enfermagem. Escola Paulista de Enfermagem - UNIFESP. São Paulo, SP, Brasil, 2016

\begin{tabular}{lcccc}
\hline Variável Interesse & Cor da pele & Renda familiar & Série & Idade \\
\hline AUDIT & & & & 0,0001 \\
$\quad$ Frequência Ingesta & 0,0114 & 0,0427 & & \\
$\quad$ QDCT & 0,0335 & & 0,0054 & \\
$\quad$ FICDV $^{+}$ & & & 0,0086 & \\
\hline
\end{tabular}

${ }^{*}$ QDCT: Quantidade de doses que consome tipicamente; †FICDV: Frequência que ingere cinco doses de uma vez

Considerando o padrão BPE, verificamos que após a $2^{\mathrm{a}} \mathrm{IB}, 29(80,6 \%)$ universitários informaram que nunca consumiam 5 doses ou mais e $7(19,4 \%)$ referiram esse consumo semanalmente ou mensalmente, frequência significantemente inferior à frequência citada na etapa anterior $p \leq 0,0418$.

A Tabela 2 mostra a regressão linear entre os escores dos domínios do AUDIT e a mobilização para mudar o padrão de consumo de bebidas alcoólicas, segundo a RPM. A maioria dos universitários que faziam uso nocivo ou provável dependência de álcool, após a $2^{\mathrm{a}}$ IB, "pensava em mudar"; os universitários que faziam uso de risco referiram que "de modo algum estavam prontos para mudar" e aqueles classificados como baixo risco estavam "preparando-se para mudar".

Tabela 2 - Regressão linear entre os domínios do AUDIT e os níveis de mobilização para mudar o padrão de consumo de álcool dos universitários de enfermagem. Escola Paulista de Enfermagem - UNIFESP. São Paulo, SP, Brasil, 2016

\begin{tabular}{|c|c|c|c|c|c|}
\hline & \multicolumn{3}{|c|}{ Resultado AUDIT - $2^{\mathrm{a}}$ IB (n/\%) } & \multirow{2}{*}{ Total } & \multirow{2}{*}{$\mathbf{p}^{\dagger}$} \\
\hline & Baixo risco & Uso risco & $\begin{array}{l}\text { Nocivol } \\
\text { PD* }\end{array}$ & & \\
\hline \multicolumn{6}{|l|}{ Diminuir padrão de consumo- $\mathrm{RPM}^{\ddagger}$} \\
\hline Não pronto para mudar & $2(8,7)$ & $2(25)$ & $0(0)$ & $4(11,1)$ & 0,0001 \\
\hline Pensando em mudar & $3(13)$ & $6(75)$ & $5(100)$ & $14(38,9)$ & \\
\hline Preparando-me para mudar & $12(52,2)$ & $0(0)$ & $0(0)$ & $12(33,3)$ & \\
\hline $\begin{array}{l}\text { Trabalhando ativamente na mudança ou a } \\
\text { mantendo }\end{array}$ & $6(26,1)$ & $0(0)$ & $0(0)$ & $6(16,7)$ & \\
\hline Total & $23(100)$ & $8(100)$ & $5(100)$ & $36(100)$ & \\
\hline
\end{tabular}

*PD: Provável dependência; †Teste da Razão de Verossimilhança, †RPM: Régua de prontidão para mudança 


\section{Discussão}

O perfil sociodemográfico dos universitários desta pesquisa demonstrou prevalência de mulheres, brancas, solteiras e com idade média de 20 anos; esse perfil assemelha-se a outros achados da literatura(11-13). Todos os universitários utilizavam álcool e a idade mediana do primeiro uso foi 15 anos. Estudo sobre uso de álcool e outras drogas realizado com universitários brasileiros evidenciou que $86,2 \%$ experimentaram álcool em alguma vez na vida e, destes, $79,2 \%$ tinham até 18 anos ${ }^{(14)}$. Em pesquisa desenvolvida com universitários do curso de enfermagem, verificou-se que $83,5 \%$ experimentaram bebidas alcoólicas e, destes, 39,5\% tinham entre 14 e 15 anos e $16,5 \%$ mais de 18 anos, quando do $1^{\circ}$ uso ${ }^{(12)}$. E para $68,1 \%$ dos universitários da área de saúde, de outro estudo, o $1^{\circ}$ uso de álcool ocorreu antes dos 17 anos ${ }^{(13)}$. A maioria dos universitários, nos diferentes estudos, revelou ter experimentado bebida alcoólica antes de 18 anos, idade de limite inferior permitido no Brasil para consumo e compra de álcool(15). Revisão integrativa de literatura evidenciou notória complexidade do tema sobre os riscos para uso nocivo/provável dependência de álcool entre adolescentes, sendo o início precoce um dos fatores de risco(16).

O tabaco e a maconha foram as SPA, exceto álcool, mais utilizadas por universitários deste estudo. Estudo nacional com universitários apurou que 46,7\% utilizaram tabaco, uma ou mais vezes na vida, e a maior prevalência foi encontrada entre universitários homens, das regiões Sul e Sudeste, com mais de 35 anos, e a SPA ilícita mais usada por eles foi a maconha com $26,1 \%{ }^{(14)}$.

No Brasil, em 2010, a bebida alcoólica mais consumida pelos adultos de ambos os sexos foi a cerveja $(61 \%)$, seguida pelo vinho $(25 \%)$ com consumo mais alto entre as mulheres e em regiões urbanas ${ }^{(17)}$. Outro estudo feito com universitários identificou semelhantes achados ${ }^{(12)}$, porém, na presente pesquisa, encontrou-se que os estudantes ingeriam mais a vodka cuja concentração de álcool é mais elevada quando comparada à concentração da cerveja e do vinho.

Pesquisa realizada na região Sul do Brasil identificou que $70,2 \%$ dos universitários ingeriam álcool em festas e churrascos, $25 \%$ na residência e $4,8 \%$ em bares e restaurantes próximos à universidade ${ }^{(13)}$.

Na Alemanha, 83,8\% dos universitários de enfermagem consumiam álcool em reuniões ou festas e $66 \%$ em discotecas ou pubs, e os motivos pelos quais bebiam eram o estresse e/ou problemas ${ }^{(18)}$.

Confirmando os motivos para o consumo de bebidas alcoólicas pelos universitários desta pesquisa, universitários de outras áreas do conhecimento, inclusive enfermagem, referiram que buscavam a confraternização e socialização por meio do consumo de álcool, por apreciarem o sabor da bebida e pela sensação de prazer e relaxamento causada pelo mesmo $^{(13)}$.

O consumo excessivo de bebidas alcoólicas pode acarretar diversos riscos e consequências, como demonstrado em pesquisa nacional com universitários que referiram $B P E$ e que vivenciaram riscos de acidentes no trânsito, intoxicação, atos de violência e abuso sexual, sexo desprotegido, comprometimento no aprendizado, comportamentos inadequados e problemas legais ${ }^{(14)}$. Em outra pesquisa, universitários da área da saúde relataram como consequências físicas do abuso da ingesta de álcool: náuseas e vômitos $(50,7 \%)$, tonturas e quedas (27,3\%), amnésia (9,9\%) e 1,4\% envolveuse em acidentes automobilísticos ${ }^{(13)}$, índices menores quando comparados às taxas de riscos sofridos por universitários desta pesquisa.

Em estudo com mulheres avaliadas pela escala Drlnc, no Centro de Atenção Psicossocial - Álcool e drogas do interior paulista, verificou-se que as consequências negativas do beber se traduziram em comprometimento em todos os aspectos avaliados, com ênfase nas áreas intrapessoal e de controle dos impulsos ${ }^{(19)}$. Tal resultado foi divergente do encontrado no presente estudo, provavelmente porque o padrão de consumo das mulheres, em tratamento, era diferente do apresentado pelos universitários investigados e associado com a menor idade dos mesmos.

Quase metade dos universitários avaliou o material didático utilizado na IB como bom e $36,1 \%$, como muito bom; assim sendo, verificamos diminuição significativa no padrão do consumo de álcool de nocivo/ provável dependente para consumidores de baixo risco $(p \leq 0,0001)$ após a segunda IB. Pesquisa realizada com universitários paulistas constatou que $56,3 \%$ do grupocontrole passaram para o padrão de baixo risco de consumo de álcool após a IB, valor um pouco menor do que deste estudo ${ }^{(20)}$, que foi de $64 \%$ após a segunda IB.

Os estudantes brancos ingeriram menos doses de bebidas alcoólicas e em menor frequência, quando comparados aos não brancos, após a segunda IB. Dados semelhantes foram encontrados em pesquisa multicêntrica realizada com a população brasileira, em 2013, que investigou o consumo de álcool nos últimos 30 dias e verificou que $13,7 \%$ dos indivíduos utilizaram álcool de forma abusiva e, destes, 47,3\% na frequência de até duas vezes ao mês, sendo maior a prevalência entre negros e pardos e na faixa etária entre 18 e 29 anos $^{(21)}$.

Nesta pesquisa, a frequência de ingesta de álcool foi menor entre estudantes com renda familiar de até 
7 SM e após a segunda IB. A relação entre o status socioeconômico individual e o consumo de álcool analisada em 33 países mostrou que as pessoas com nível socioeconômico mais elevado eram mais propensas ao uso do álcool, enquanto o BPE esteve associado a homens com nível socioeconômico mais baixo. Entre as mulheres, o consumo de risco correlacionou-se ao maior nível socioeconômico, principalmente nos paises de média e baixa renda ${ }^{(22)}$. Ocorreu resposta positiva da IB para os estudantes do primeiro ano $(p \leq 0,0086)$, os quais relataram ter diminuído a frequência da ingesta, uma vez que, possivelmente, ainda não estavam muito influenciados pelos novos hábitos e pelas festas. $O$ consumo aumentado de 5 ou mais doses, por episódio, correspondeu a $32,1 \%$ dos universitários de enfermagem cariocas nas diferentes séries do curso(12). No presente estudo, identificou-se diferença significativa no número de doses consumidas entre as séries, assim como diminuição da frequência do BPE após a segunda IB, dados também encontrados em outra pesquisa que realizou IB com universitários ${ }^{(20)}$.

Estudo longitudinal que utilizou a RPM com suíços, abusadores e dependentes de álcool encontrou pontuação mais alta na prontidão e confiança para decisão de parar de beber e manter abstinência e menor pontuação para a importância de mudar o comportamento de beber ${ }^{(23)}$. Norte-americanos entre 21 e 75 anos, classificados como consumidores abusivos ou dependentes de álcool, acompanhados em serviços de atenção primária, receberam IB por enfermeiras e na sequência demonstraram prontidão para mudança e $36 \%$ informaram ser importante mudar o comportamento do consumo de álcool; $44,1 \%$ estavam muito confiantes na capacidade de mudar; e 42,8\% mais ou menos prontos para mudar ${ }^{(24)}$. Neste estudo, $52,2 \%$ dos universitários de enfermagem, que após a $2^{\mathrm{a}} \mathrm{IB}$ estavam no padrão do baixo risco do consumo de álcool, relataram, por meio da RPM, que estavam prontos para mudar o padrão de consumo de álcool e $75 \%$ dos que estavam no padrão de uso de risco referiram estar apenas pensando em mudar.

\section{Conclusão}

Mulheres brancas, com religião e renda familiar mensal mediana predominaram entre os bebedores de risco/provável dependência. A associação do consumo de álcool e outras SPA foi demonstrada. A idade mediana do início do uso de bebida alcoólica foi baixa, o que pode gerar consequências futuras importantes. Riscos e consequências associadas ao consumo de álcool foram relatos frequentes e com variados graus de perigo que puseram a segurança do universitário em risco.
Provavelmente, o escore do Drlnc sofreu influência da idade média, ainda baixa, dos alunos e do padrão de consumo.

A IB surtiu efeito positivo e significativo no padrão de consumo de álcool e na predisposição para a mudança dos universitários de enfermagem.

Medidas de prevenção do uso e abuso de álcool devem ser programadas, em especial, para neutralizar ou minimizar as correlações entre achados do AUDIT e variáveis sociodemográficas e econômica visando à prevenção de problemas decorrentes do consumo excessivo do álcool e promoção da saúde física e psicossocial.

\section{Agradecimentos}

Agradecemos a todos os estudantes que participaram da pesquisa e aos docentes que cederam períodos das aulas para coleta de dados e realização da Intervenção Breve.

\section{Referências}

1. World Health Organization. Global status report on alcohol and health: 2014 [Internet]. Geneva: World Health Organization; 2014 [cited 2017 Jun 10]. 392 p. Available from: http://apps.who.int/iris/bitstre am/10665/112736/1/9789240692763_eng.pdf

2. Pan American Health Organization. Regional status report on alcohol and health in the Americas [Internet]. Washington (DC): Pan American Health Organization; 2015 [cited 2017 Jun 10].80 p. Available from: http://www2. paho.org/hq/index.php?option=com_content\&view=article $\& i d=11108$ \&ltemid $=41530$ \&lang $=e n$

3. Nair JM, Nemeth LS, Sommers M, Newman S, Amella E. Alcohol use, misuse, and abuse among nursing students: a photovoice study. J Addict Nurs. 2016 Jan-Mar; 27(1):12-23. 4. Malta DC, da Silva JB Jr. [Brazilian strategic action plan to combat chronic non-communicable diseases and the global targets set to confront these diseases by 2025 : a review]. Epidemiol Serv Saúde. [Internet]. 2013 Jan-Mar [cited 2017 Jun 13];22(1):151-64. Portuguese. Available from: http://scielo.iec.pa.gov.br/pdf/ess/v22n1/v22n1a16. pdf

5. Beaglehole R, Bonita R, Horton R, Ezzati M, Bhala N, Amuyunzu-Nyamongo $\mathrm{M}$, et al. Measuring progress on NCDs: one goal and five targets. Lancet. 2012 Oct 13; 380(9850):1283-5.

6. DiFulvio GT, Linowski SA, Mazziotti JS, Puleo E. Effectiveness of the Brief Alcohol and Screening Intervention for College Students (BASICS) program with a mandated population. J Am Coll Health. 2012; 60(4):269-80. 
7. Pereira MO, Anginoni BM, Ferreira $\mathrm{N}$ da $\mathrm{C}$, de Oliveira $M A$, de Vargas D, Colvero $L$ de A. [Effectiveness of the brief intervention for the use of abusive alcohol in the primary: systematic review]. Rev Bras Enferm. [Internet]. 2013 MayJun [cited 2017 Jun 10];66(3):420-8. Portuguese. Available from: http://www.scielo.br/pdf/reben/v66n3/a18v66n3.pdf 8. Lima CT, Freire AC, Silva AP, Teixeira RM, Farrell M, Prince M. Concurrent and construct validity of the audit in an urban Brazilian sample. Alcohol Alcohol. [Internet]. 2005 Nov-Dec [cited 2017 Jun 10];40(6):584-9. Available from: https://academic.oup.com/alcalc/article-lookup/doi/10.1093/ alcalc/agh202

9. Figlie NB. Motivação em alcoolistas tratados em ambulatório específico para alcoolismo e em ambulatório de gastrenterologia: dados do projeto piloto [Dissertation]. São Paulo: Universidade Federal de São Paulo; 1999. 10. Velásquez M, Maurer G, Crouch C, Di Clemente C. Group treatment for substance abuse: a stages-of-change therapy manual. New York: The Guilford Press; 2001.

11. da Silva BP, Corradi-Webster CM, Donato EC, Hayashida M, de Siqueira MM. [Common mental disorders, alcohol consumption and tobacco use, among nursing students at a public university in the Western Brazilian Amazon]. SMAD, Rev Eletrônica Saúde Mental Álcool Drog. [Internet]. 2014 May-Aug [cited 2017 Jun 10];10(2):93-100. Portuguese. Available from: http://www.revistas.usp.br/smad/article/ view/98724/97287

12. Tavares-Jomar R, dos Santos-Silva E. [Consumption of alcoholic beverages among nursing students]. Aquichan. [Internet]. 2013 May-Aug [cited 2017 Jun 10]; 13(2):22633. Portuguese. Available from: http://www.scielo.org.co/ pdf/aqui/v13n2/v13n2a09.pdf

13. Baumgarten LZ, Gomes VL, da Fonseca AD. [Alcohol consumption among university students in the health area of Federal University of Rio Grande/RS: subsidy to the nursing]. Esc Anna Nery. [Internet]. 2012 Sep [cited 2017 Jun 10];16(3):530-5. Portuguese. Available from: http:// www.scielo.br/pdf/ean/v16n3/15.pdf

14. Presidência da República (BR). Secretaria Nacional de Políticas sobre Drogas. I Levantamento Nacional sobre o uso de álcool, tabaco e outras drogas entre universitários das 27 capitais brasileiras [Internet]. GREA/ IPQ-HCFMUSP; de Andrade AG, Duarte P do C, de Oliveira LG, organizadores. Brasília: SENAD; 2010 [cited 2017 May 20]. 282p. Available from: http://www.grea.org.br/userfiles/ GREA-ILevantamentoNacionalUniversitarios.pdf

15. CISA: Centro de Informações sobre Saúde e Álcool [Internet]. São Paulo: CISA; 2017. Álcool e jovens; [cited 2017 Jun 13]; [about 1screen]. Available from: http://www. cisa.org.br/artigo/340/alcool-jovens.php

16. Rozin L, Zagonel IP. Risk factors for alcohol dependence in adolescents. Acta Paul Enferm. [Internet]. 2012 [cited
2017 Jun 13]; 25(2):314-8. Available from: http://www. scielo.br/pdf/ape/v25n2/en_a25v25n2.pdf

17. Laranjeira R, Pinsky I, Sanches M, Zaleski M, Caetano R. Alcohol use patterns among Brazilian adults. Rev Bras Psiquiatr. [Internet]. 2010 Sep [cited 2017 Jun 13]; 32(3):231-41. Available from: http://www.scielo.br/pdf/rbp/ v32n3/aop1209.pdf

18. Lehmann F, von Lindeman K, Klewer J, Kugler J. BMI, physical inactivity, cigarette and alcohol consumption in female nursing students: a 5-year comparison. BMC Med Educ. [Internet]. 2014 Apr 17 [cited 2017 Jun 13]; 14:82. Available from: https://www.ncbi.nlm.nih.gov/pmc/articles/ PMC3997793/pdf/1472-6920-14-82.pdf

19. Pillon SC, dos Santos MA, Florido LM, Cafer JR, Ferreira PS, Scherer ZA, et al. [Consequences of alcohol consumption among women cared for in a Psychosocial Care Center]. Rev Eletron Enferm. [Internet]. 2014 Apr-Jun [cited 2017 Jun 13]; 16(2):338-45. Portuguese. Available from: https://revistas.ufg.br/fen/article/view/22712/17034 20. Silva EC, Tucci AM. [Brief intervention to reduce alcohol consumption and its consequences in brazilian university students]. Psicol Reflex Crit. [Internet]. 2015 Oct-Dec [cited 2017 Jun 10]; 28(4):728-36. Portuguese. Available from: http://www.scielo.br/pdf/prc/v28n4/01027972-prc-28-04-00728.pdf

21. Garcia LP, de Freitas LR. Heavy drinking in Brazil: results from the 2013 National Health Survey. Epidemiol Serv Saúde. [Internet]. 2015 Apr-Jun [cited 2017 Jun 13]; 24(2):227-37. Available from: http://www.scielo.br/pdf/ress/ v24n2/en_2237-9622-ress-24-02-00227.pdf

22. Grittner U, Kuntsche S, Gmel G, Bloomfield K. Alcohol consumption and social inequality at the individual and country levels: results from an international study. Eur J Public Health. [Internet]. 2013 Apr [cited 2017 Jun 13]; 23(2):332-9. Available from: https://www.ncbi.nlm.nih.gov/ pmc/articles/PMC3610336/pdf/cks044.pdf

23. Gaume J, Bertholet N, Daeppen JB. Readiness to change predicts drinking: findings from 12-month follow-up of alcohol use disorder outpatients. Alcohol Alcohol. 2017 Jan; 52(1):65-71.

24. Bradley KA, Ludman EJ, Chavez LJ, Bobb JF, Ruedebusch SJ, Achtmeyer CE, et al. Patient-centered primary care for adults at high risk for AUDs: the Choosing Healthier Drinking Options In primary Care (CHOICE) trial. Addict Sci Clin Pract. [Internet]. 2017 May 17 [cited 2017 Jun 13]; 12(1):15. Available from: https://www.ncbi. nlm.nih.gov/pmc/articles/PMC5436432/pdf/13722_2017_ Article_80.pdf

Recebido: 29.08.2017 Aceito: 31.07 .2018 